\title{
Selection and evaluation of Internet information by adults with intellectual disabilities
}

Dr. Ladislao Salmerón ${ }^{1}$, Dr. Inmaculada Fajardo ${ }^{1}$, Dr. Marcos Gómez-Puerta ${ }^{2}$

${ }^{1}$ Department of Developmental and Educational Psychology, University of Valencia, Valencia, Spain.

${ }^{2}$ Department of Developmental Psychology and Didactics, University of Alicante, Alicante, Spain.

European Journal of Special Needs Education

Manuscript in press, as of 20/04/2018

Correspondence:

Dr. Ladislao Salmerón

University of Valencia

Avda. Blasco Ibáñez, 21

46010 Valencia, Spain

ladislao.salmeron@uv.es 


\title{
Selection and evaluation of Internet information by adults with intellectual disabilities
}

\begin{abstract}
Internet offers people with intellectual disabilities (ID) unique opportunities to access information and to participate in society. But concerns have been raised about the potential risks they face when accessing the Internet (e.g. giving credit to false information, being exposed to manipulative content). As part of the current debate between positive risk-taking and overprotection, our study empirically tested the extent to which 43 adults with ID identified and selected topically relevant as well as trustworthy web pages while searching the Internet for several topics (e.g. Can socialnetworks use your pictures for advertisement?). Participants also justified their search decisions. Results revealed that while searching familiar topics (i.e. social networks), participants selected more relevant and trustworthy pages than irrelevant and less trustworthy ones. Searches of less familiar topics (i.e. daily health), were carried out randomly, that is, without applying a specific criterion. Results point to the importance of topic familiarity on people's with ID performance on Internet searching tasks. This pattern of results suggests that, first, we should avoid overprotection when people with ID search for familiar topics and, second, they need more support when searching for information about less familiar topics.
\end{abstract}

Keywords: intellectual disabilities; Internet risks; Internet search; trustworthiness; positive risk-taking.

\section{Introduction}

The use of the Internet has become in our society a basic tool for consulting information, interacting socially or accessing certain online services, among other alternatives. Accordingly, digital literacy and Internet access are key elements to avoid the digital exclusion of certain groups such as people with intellectual disabilities (ID). Although Internet accessibility for these people continues to show difficulties (e.g. 
cognitive accessibility to online information), its use has grown significantly in recent years (Chadwick, Wesson and Fullwood 2013); nevertheless, this continues to be below the general population (Caton and Chapman 2016).

Not all barriers to Internet use by this group are related to accessibility. Prejudices towards people with ID in the real world (World Health Organization 2011) seem to be also manifesting also online (Chadwick and Wesson 2016). One of the prejudices that underlie the overprotection of this group is the perception by caregivers (e.g. parents, therapists, teachers) of a greater susceptibility to being abused. In this sense, it is presumed that if they are vulnerable in real life, then they will as well be highly vulnerable online (Buijs et al. 2017). This situation is derived from a greater perception of potential risks (e.g. investing money, being sexually abused, giving credit to false information or being exposed to manipulative content) on the part of the caregivers in the use of the Internet that people with ID make (Chadwick, Quinn, and Fullwood 2017; Lough and Fisher 2016; Seale and Chadwick 2017). In this way, the potential benefits of its use can be mediated by the caregivers' fears that these people have problems online.

The studies carried out about use of the Internet by people with ID and the perception of the benefits and risks associated with it are still very scarce (Normand and Sallafranque-St-Louis 2016), although we note below certain trends identified so far.

\section{Benefits and risks of Internet use for people with ID}

On one side, specific benefits have been identified for people with ID in their use of the Internet such as social interaction, access to support groups (Molin, Sorbring, and Löfgren-Martenson 2015), self-determination, learning and entertainment (Chadwick et al. 2013). The studies show that the device most used by them to access the Internet is the smartphone (Didden et al. 2009; Gutiérrez and Martorell 2011) and that the activities they usually do online are watching videos, chatting with friends, or reading and writing on social networks (Chiner, Gómez, and Cardona 2017a).

On the other hand, previous empirical literature and clinicians' reports suggest that Internet risks may be significant for individuals perceived as vulnerable such as children (Livingstone, Haddon, Görzig, and Ólafsson 2011) or persons with ID. In this second case, in a study conducted in the Spanish context (Chiner, Gómez, and Cardona 2017a), 77 adults with ID reported that they had encountered several problems in their 
use of the Internet, among them: losing money by bets or non desired buys (89\%), someone tried to sell them something (62\%), someone sent them non-desired pictures or videos with sexual content (56\%), or someone threatened them (53\%). Complementing these data, these same authors (2017b) found through a study carried out with 68 caregivers (i.e. parents, professionals at support centers) of people with ID that there is the perception that the Internet is an unsafe $(21 \%)$ or very unsafe $(75 \%)$ environment for children with ID. Moreover, this perception is maintained in the case of adults with this disability considering the Internet as unsafe $(49 \%)$ or very unsafe $(42 \%)$ for them. Caregivers also felt as unprepared to prevent these problems $(60 \%)$ or to deal with them (53\%). Participants identified as their main concerns someone using the personal information of the individual with ID without his or her consent (66\%), being asked for information or photos (64\%), receiving sexual photos or videos that they do not want to see $(61 \%)$, being threatened $(59 \%)$, or someone telling others unpleasant things about them $(50 \%)$. In addition to this view, the media tends to focus on the risks arising from the Internet and easy access to inappropriate content (Löfgren-Mårtenson 2008), raising concerns of caregivers about how people with ID should use the Internet.

This perception of caregivers about the vulnerability online of people with ID is especially relevant since, as Palmer et al. stated (2012), family members and professionals are the main support for people with ID in the use of technologies. Generally, caregivers show strong feelings of responsibility towards the care and behaviour of the individual with ID. This attitude affects the actions of caregivers, showing a tendency to exercise greater control over the online behavior of people with ID, which manifests itself in online overprotection behaviors such as limitations or restrictions on Internet use or more control over online activities (Löfgren-Mårtenson 2008). In this sense, if the caregivers perceive themselves as not very competent to face certain potential risks that people with ID can experience online, they tend to offer them fewer opportunities to access the Internet (Chadwick and Wesson 2016). Overall, the perception of potential risks as well as the tendency to online overprotection of this group by caregivers can significantly limit the development of digital competence and become a subtle form of discrimination through various levels of digital exclusion.

Faced with this attitude of overprotection, the positive risk taking approach (Perske 1972) understands that risks are an inherent part of the lives of people with and without disabilities and that, therefore, training for their coping or resolution is necessary for our development as human beings. This approach promotes shared 
decision-making between people with ID and their caregivers regarding the risks that are acceptable and which actions should be developed to avoid problems (McConkey and Smyth 2003). As highlighted by Seale (2014), this strategy could be an appropriate approach for the management of online risks by people with ID. However, there are studies (Clarke et al. 2005) that have shown that the divergence in the perception of the degree of risk between caregivers and people with ID is a factor that can significantly limit the joint decision-making. Anyway, as Chadwick et al. (2017) pointed, if they are actually more vulnerable then prevention and support programs should be designed for improving online protection.

In sum, one important question is to determine the optimal contexts in which people with ID can safely take the risk to select information on the Internet. Determining safer contexts may allow fostering self-determination by means of positive risk-taking (Alaszewski and Alaszewski 2002; Seale 2014).

\section{Selection and evaluation of information on the Internet}

To successfully confront the risks of searching information on the Internet stated above people must consider both semantic and trustworthiness cues of the web pages (Salmerón et al. 2013). On the one hand, semantic cues allow people to identify to what extent a web page is relevant for his/her searching purposes. People tend to use two semantic cues: superficial (i.e. words in the web page title that literally match their current search) and deep processing (i.e. semantic overlap between the searching and web page title). A major developmental shift occurs during adolescence, when students abandon superficial word cues as their main resource, and start using deep processing cues (Keil and Kozminsky 2013). The use of deep processing cues is contingent upon having high comprehension skills (Salmerón et al. 2015). Thus, to the extent that students with ID generally have poor comprehension skills (Fajardo et al. 2013, 2014), we may expect that students with ID may show a developmental delay in the use of deep processing cues to select topically relevant pages. Other personal characteristics,

such as topic familiarity, can help students to overcome comprehension difficulties to select relevant pages based on deep processing rather than on superficial cues (Rouet et al. 2011).

On the other hand, trustworthiness cues let people quickly evaluate the quality of the information provided in a web page, which frequently can be deceiving, either 
because the author lacks the necessary competence to write about the topic or because she has vested interests (e.g. commercial, ideological) other than to inform in a neutral way. The ability to identify and use trustworthiness cues during Internet searching has been less documented, but related research on the evaluation of information in social media suggests that it also develops through adolescence (Salmerón et al. 2016b). In a related study, we found that students with ID didn't consider the competence of author's message when recommending messages in Internet forums (Salmerón et al. 2016a), contrary to what was found in adolescents with typical development.

In this research, we test if the online vulnerability presumption is accurate regarding a particular aspect of online behavior: evaluation of Internet searching results. In individuals with typical development, a strong developmental shift during adolescence in evaluations of Internet searching results has been described. To identify and select potentially relevant web pages, such a shift consists of changing from the usage of superficial cues like lexical matching (e.g. select a result that includes a word matching the request), to the application of semantic inferences (e.g. select a result which is semantically relevant, regardless of the words used) (Keil and Kozminsky 2013). Therefore, we tested the existence of a developmental delay in the acquisition of deep cues, and if such delay was moderated by the type of topics searched. The results would be an empirical support to the assumption that, under some topics, people with ID are more vulnerable to online risks, such as selecting a page irrelevant for their goals, or one authored by an untrustworthy source.

\section{Rationale for the present study}

We tested to what extent adults with ID select relevant and trustworthy pages during Internet searching. To test this, we developed a series of Internet searching scenarios on different topics. Each scenario presented a list of four results, all containing a critical word in the searching query. Thus, in order to select a topically relevant web page, participants must deeply process the context of the web page title and snippet. In addition, each web page included explicit trustworthy information in the URLs. Based on our review of the literature that suggest a developmental delay, our hypothesis 1 stated that across all searches adults with ID would not efficiently select the most relevant and trustworthy pages. 
In addition, we tested if such delay was moderated by the familiarity with the searched topics. Previous literature has found that people with ID are particularly interested in the topic of technology, and specifically on the use of computers to access information on web pages or social media (Lloyd et al. 2006). Following previous studies (Rouet et al. 2011), we expected that adults with ID would be more efficient in selecting information in topics for which they have more interests in, for which they may have developed a richer knowledge base. Specifically, our hypothesis 2 stated that adults with ID select more relevant and trustworthy pages for more familiar topics (i.e. social networks) than for less familiar topics (i.e. daily health). In the same line, our hypothesis 3 stated that adults with ID use more complete and topic oriented justifications for more familiar than less familiar topics.

\section{Method}

\section{Design}

This study was carried out according to a quantitative approach and quasi-experimental method, following a within-groups design. The experiment had an independent variable (IV) consisting of the presentation of Google search-engine result pages (SERPs). Specifically, two elements were manipulated in this variable: topic relevance (high / low topic relevance) and page trustworthiness (high / low page trustworthiness), generating in the SERP four levels of search results: (a) high topic relevance-high page trustworthiness, (b) high topic relevance-low page trustworthiness, (c) low topic relevance-high page trustworthiness, and (d) low topic relevance-low page trustworthiness. The dependent variables (DV) were the choice of the web page to obtain the necessary information by the participants, as well as the justification for that choice. Below we detailed the manipulation of the IV and the measurement of the DVs.

The Ethical Committee of Experimental Research of the University of Valencia approved the study (procedure H1443008998347), according to the principles of the Declaration of Helsinki.

\section{Participants}

The study focused on the population available in two vocational centres for adults with ID of the Valencian Community (Spain). Attendants to these centres participate in long training programs for access to employment (with a duration of between one and two 
years), especially designed for people with ID; to participate in these training actions, individuals must meet the following criteria: (1) have an official diagnosis and certificate of ID, (2) have completed the 10 years of schooling which are mandatory in Spain, and (3) have enough personal and social autonomy to follow different job training modules. The population of these centres was composed of 57 adults with ID. Of these, 47 had mild ID (82.5\%) and 10 moderate ID (17.5\%). The level of ID was taken from their official disability certificate, in which the Government itself indicates that level according to the diagnosis made previously. The sample was recruited to participate after consultation and approval of the centre's pedagogical team. Also, the informed consent of participants was collected and they were given the option to withdraw from the study at any time, if they so wished. Of the total of 47 people with official certificate of disability, a convenience sample of 43 agreed to participate in the study (92.5\%). Of them, 39.5\% were female and $60.5 \%$ were male; their average age was 22.9 years $(\mathrm{SD}=3.93$, ranging from 18 to 33 ). Its average verbal IQ was $72+-8$ which corresponds to mild severity level. This value was calculated for the $63 \%$ of the sample (27 out of 43) based on the Vocabulary subtest's scores of the K-BIT (Cordero and Calonge 2000) that the vocational centers provided. They adopted the study as a school activity aimed to foster digital media use in classrooms. Most participants $(n=$ $39 / 90.7 \%$ ) reported a daily use of the Internet outside the centre, either with smartphones (93\%) and/or computers (69.7\%). They used the Internet mostly to participate in social networks and to watch videos on Youtube.

\section{Instruments}

The instrument used for data collection was the questionnaire called Ski-III, which was adapted from a tool developed in a recent project aimed at fostering adolescents' critical evaluation of Internet information (Pérez et al. 2018). It was previously piloted in a group of 8 adults with ID who attended a sheltered workshop center. Thanks to this we were able to improve the instrument according to their perceptions and tips such as including an example before the questions, explaining the instructions and example using a Powerpoint presentation to offer visual support, or highlighting the questions in a box. 
The questionnaire consisted of five sheets: instructions and example sheet to train the participants, and eight questions (two in each remaining sheet) that had to be autonomously answered.

\section{Search-engine results pages}

The instrument presented four Google search-engine results pages (SERPs) on topics related to social networks ('Lingo: a new social network' and 'Privacy of your pictures in Facebook'), and to daily health issues ('Risks of following a vegetarian diet' and 'Using sunbath to get tanned'). Each SERP included four links which had in their titles the target words for each search (e.g. 'pictures' and 'Facebook'). Each link resulted from crossing (1) topic relevance (with two levels of relevance: [a] high: the title included information that could be relevant to the topic, and [b] low: the title didn't include information relevant, although it included the target words), and (2) page trustworthiness (with two levels of trustworthiness: [a] high: the URL was from an institution, such as the police, or a hospital, and [b] low: the URL showed personal and non-institutional pages). Similar to the procedure used by Keil and Kozminsky (2013), each SERP was presented on a single questionnaire sheet which had been printed in color to make it as similar as possible to the Google website, showing the links and URL in the colors they had shown on the original page (see Figure 1). We designed eight versions of the questionnaire by randomizing, on the one hand, the order of presentation of the questions (and, therefore, the topics) and, on the other hand, the results of the Google search for each topic. This was done to control possible response biases related to the order of presentation of the topics and / or results.

-Insert Figure 1 about here or include it as an annex -

On the first question of each SERP, students had to indicate in their responses which web page they would visit to obtain adequate information on the topic of the question, by marking the selected page with a cross next to it. Then, on the second question of each SERP, students had to write down a justification on why they considered that the web chosen was the best one to obtain the information they needed to answer the question related to the exposed topic.

We classified the answers through content analysis, grouping the justifications in the following categories, according to the type of argument used: related to the topic, 
related to the reliability of the source or an argument out-of-focus (cf. Salmerón et al. 2016b). Examples for each category are given in table 1. Specifically, we used the following rubric: a) 'topic justification' - the response identifies a page that can provide useful information to their query; b) 'trust justification' - the response explicitly mentions that the page is secure, trustworthy, or reliable; or c) 'out-of-focus' - the response contains other topics not addressed in the query, or students' opinion, without any attempt to reconcile the claim with the actual query (cf. Van Nieuwenhuijzen et al. 2011). Two raters coded the responses from a subsample of 22 students and obtained good inter-rater agreement (Cohen's kappa $=.80)$. Disagreements were resolved via discussions.

-Insert Table 1 about here-

\section{Procedure}

A member of the research group went to the headquarters of the training center from which the sample was obtained. The pass of the test was made in the classroom of the group of participants. First, the purpose of the study and the type of questionnaire that was to be applied were explained. Also, the informed consent was read and they were given a few minutes to think if they wanted to participate in the study. Those people who decided to participate signed the informed consent. The paper questionnaire was then distributed to the participants (alternating the different versions of the questionnaire according to the order of the questions and possible answers) and the initial example of the questionnaire was carried out in group, in order to ensure that they understood the task. This activity was carried out by also projecting the question through a Powerpoint presentation. Questions were answered until we were sure that everyone had understood the activity. Then, each student individually carried out the questionnaire autonomously and at their own pace. A member of the research team supervised the whole process, verbally supporting those people who had some doubt about how to proceed with their answers. Altogether, the process lasted approximately for 45 minutes.

\section{Results}


First, we tested our hypothesis 1 (i.e. adults would not efficiently select the most relevant and trustworthy pages in Internet results). For each participant we calculated how many of their selections corresponded to each of the four available links (high or low topic relevant, and high or low trustworthiness), across the four search topics. Friedman test revealed that there were significant differences $\left(\chi^{2}(3, N=43)=14.41, p=\right.$ .002). Contrary to our expectations, across the four searched topics students with ID selected more often the high topic relevant and high trustworthy result $(M=38.37 \%, S D$ $=29.05$ ), than the other types of results (high topic relevant and low trustworthy: $26.75 \%, S D=24.04$; low topic relevant and high trustworthy: $12.79 \%, S D=18.40$; low topic relevant and low trustworthy: $22.09 \%, S D=24.52$ ).

Second, we tested our hypothesis 2 , stating that adults with ID will select more relevant and trustworthy pages for well-known topics (i.e. social networks) than for less know topics (i.e. daily health). To do so, we calculated the percentage of students that selected each of the four available links (high or low topic relevant, and high or low trustworthiness). We analyzed the results individually for each topic. Independent Chi square analyses indicated that participants' selection of web pages differed significantly for the social networks topics, $\left(\chi^{2}(3, N=43)=13.28, p=.004\right.$, and $\chi^{2}(3, N=43)=16.81, p$ $=.001$, for the Lingo and Facebook topics, respectively). As can be seen in table 2, the majority of adults with ID selected the high topic relevant and high trustworthy page, rather than the other pages. By contrast, participants' selection of pages for the daily health topics didn't differ as a function of page type $\left(\chi^{2}(3, N=43)=3.05, p=.385\right.$, and $\chi^{2}(3, N=43)=3.98, p=.26$, for the vegetarian diet and sunbath cabins topics, respectively).

-Insert table 2 about here-

Third, we tested our hypothesis 3 stating that adults with ID use more complete and longer justifications for well-known topics (i.e. social networks) than for less know topics (i.e. daily health). In general, participants tended to write rather short justifications, which prevented us from running an analyses based on the number of ideas included. Instead, we first did a content analysis based on the number of words in students' justification for their page selection. $T$ tests were used to compare the justification of each topic with the other three. As can be seen in Table 3, students' wrote longer justifications for the Facebook topic than the other three, but the difference 
was significant only for the comparison against the sunbath cabins, $t(42)=2.45, p=$ .018 , and the Lingo social network, $t(42)=2.48, p=.017$, but not for the vegetarian diet, $t(42)=1.22, p=.229)$. None of the other comparisons resulted in significant differences (all $t \mathrm{~s}<1.1$ ). Thus, the analysis of justification length provided only partial support to our hypothesis 3 .

Finally, we further tested hypothesis 3 by doing a content analysis comparing the type of justifications (topic, trust, or out-of-focus justification) in each of the four topics. As can be seen in table 3, in all four topics the majority of students used out-offocus justifications. A quarter of students used topic justifications, and they seldom used trust justifications. Most critical for our hypothesis, we compared the responses across the four topics using the Friedman test. Results indicated that the type of justifications didn't differ across topics, $\chi^{2}<1$.

-Insert table 3 about here-

\section{Conclusions}

Results from our study provide novel insights about how adults with mild ID select web pages from the Internet. Contrary to pessimistic views on the literacy skills of adults with mild ID, the results revealed that these individuals are able to select relevant and trustworthy web pages of topics on technology issues, while discarding distractor pages including either irrelevant information or less trustworthy sources. For the searches involving health topics, their selection of pages was rather random, as they selected both relevant and irrelevant pages, as well as more or less trustworthy ones. As such, our results do not support the existence of an important difference compared to adults without ID in the ability to select relevant information on the Internet, at least provided that they are searching an interesting and familiar topic (i.e. social networks).

A critical aspect of the results was the different role played by the topic being searched. Computer technology is a generally interesting topic for young people with ID (Lloyd et al. 2006). This interest may have boosted their use of such technologies and the acquisition of a rich knowledge base on the topic. Participants may have used their knowledge and Internet search experience to identify topically relevant web pages for 
their search, and to discard appealing but not topically relevant ones (as all webpage titles included a literal keyword from their search)(cf. Rouet et al. 2011). In addition, it is probable that previous experience with the topics may have increased their chances to get exposed to critical situations such as accessing inappropriate content (Chiner et al. 2017a), which could have raised their attention to sources. This may partially explain their sensitivity to not only topically relevant but also trustworthy web pages, in the technological topics.

Regarding health-related issues, a possible explanation for the worst selection of these sources could be related to the possibility that this issue is not in the interest of these people (and therefore they do not look for information on the Internet) or those are not subjects of which they often take care by themselves, delegating these issues to their relatives or support professionals. Therefore, the selection strategies applied to the topics related to technologies were not used in this case, probably due to lack of specific experience in the search and selection of Internet sources on health issues. Unfortunately, participants' responses in the justification task didn't shed light on the processes they undertook to evaluate the Internet results in our study. Thus, our explanations are tentative and should be interpreted with caution.

In a majority of cases adults with mild ID could not justify their page selections, and instead they tended to write justifications that range outside the focus of their search query. In this same line, Salmerón et al. (2016a) reported that students with ID tended to include opinions to justify why they recommend or not suggestions in a web forum, without any attempt to link such opinions to the discussion held in the forum. Such pattern could be due to the fact that adults with ID tend to have difficulties with writing expression. When people with ID are given the opportunity to participate in writing workshops that include mini-lessons on writing skills, and several opportunities for writing practice and reflection on self-selection of topics, they can improve their writing skills (Sturm 2012). Linking their writing activities to authentic reading activities such as searching the web could be a fruitful way to enrich the literacy skills of students with ID (Copeland and Keefe 2017), and could help to gain a deeper understanding of their mental processes in complex literacy tasks such as Internet search.

\section{Educational implications}


We concur with Morgan et al. (2011) in that we must increase our understanding about the literacy practice that adults with ID carry in their daily lives, as they may be more proficient than in more traditional school-based literacy tasks. Based on our results, practitioners could start their interventions to teach Internet search using familiar topics for which adults with ID had already background knowledge, and for which they may feel confident enough. Similarly, caregivers could promote risk-taking behaviors in the context of web search for familiar topics.

\section{Limitations}

For this study we created a search scenario in paper that mimicked the first step of an Internet search (cf. Keil and Kozminsky 2013). Although at first sight this scenario can be perceived as highly unnatural, it allowed us to uniquely control for several potential confounding factors, such as the position of the link on the list of results, or the appealing of the URL. Future studies could extend this research to more natural environments, such as introducing Internet search tasks in the school computer lab. In addition, online measures such as eye-tracking could be introduced to clearly identify the ways in which results are processed (for recent eye-tracking studies with people with ID see Tavares et al. 2015). Likewise, future works could replicate this study by expanding and diversifying the issues raised, for example, including topics on which participants have more knowledge or others on which they may have more interest (e.g. travel, job searches, sexuality). Also, a previous analysis of the familiarity of each of the topics in Internet searches for the participants could be addressed, in order to be able to control this possible extraneous variable in the study.

Regarding trustworthiness, this study applied the criterion that the information from institutional websites (e.g. police, government) should be prioritized by participants in their choices because, in principle, these sources could provide more reliable and objective information than other web pages. However, this aspect is debatable since even such information should be evaluated in itself and every Internet user should verify if said criteria were specifically met. Therefore, this question should be adequately explored in future experiments.

Regarding the justification of the choice of the information source, future research could conduct and record semi-structured interviews and make a qualitative analysis of these data. This approach could provide valuable and more detailed 
information about the reasons for choosing the website in relation to the perception of the trustworthiness of the source and the relationship with the topic.

At this point, our study offers new methods and new insights to understand how people with ID search for information on the Internet, and suggests we should avoid overprotection when they search for familiar topics.

Funding. The Ministry of Education, Culture, and Sports (General Secretariat of Universities) supported this work under Grant number EDU2014-59422P.

Disclosure statement. The authors reported no potential conflict of interest.

Acknowledgements. Researchers would like to thank participants for their generous contribution to this study and to Asociación APSA for the facilities offered to carry out the research.

\section{References}

Alaszewski, A., and H. Alaszewski. 2002. Towards the Creative Management of Risk: Perceptions, Practices and Policies. British Journal of Learning Disabilities 30 (2): 56-62.

Buijs, P., E. Boot, A. Shugar, W. L. A. Fung, and A. S. Bassett. 2017. Internet Safety Issues for Adolescents and Adults with Intellectual Disabilities. Journal of Applied Research in Intellectual Disabilities 30: 416-418.

Caton, S. and M. Chapman. 2016. The Use of Social Media and People with Intellectual Disability: A Systematic Review and Thematic Analysis. Journal of Intellectual and Developmental Disability 41: 125-139.

Chadwick, D. D., S. Quinn, and C. Fullwood. 2017. Perceptions of the Risks and Benefits of Internet Access and Use by People with Intellectual Disabilities. British Journal of Learning Disabilities 45 (1): 21-31.

Chadwick, D., and C. Wesson. 2016. Digital Inclusion and Disability. In Applied Cyberpsychology, edited by A. Attrill and C. Fullwood, 1-23. London: Palgrave Macmillan 
Chadwick, D., C. Wesson, and C. Fullwood. 2013. Internet Access by People with Intellectual Disabilities: Inequalities and Opportunities. Future Internet 5 (3): 376-397.

Chiner, E., M. Gómez-Puerta, and C. Cardona-Moltó. 2017a. Internet Use, Risks and Online Behavior: The View of Internet Users with Intellectual Disabilities and their Caregivers. British Journal of Learning Disabilities 45: 190-197.

Chiner, E., M. Gómez-Puerta, and C. Cardona-Moltó. 2017b. Internet and People with Intellectual Disability: An Approach to Caregivers' Concerns, Prevention Strategies and Training Needs. Journal of New Approaches in Educational Research 6 (2): 153-158. doi:10.7821/naer.2017.7.243

Clarke, C. L., M. Lhussier, C. Minto, C. E. Gibb, and T. Perini. 2005. Paradoxes, Locations and the Need for Social Coherence: A Qualitative Study Of Living with a Learning Difficulty. Disability and Society 20 (4): 405-419. doi:10.1080/09687590500086534

Cordero, A., and I. Calonge. 2000. Test breve de inteligencia de Kaufman (K-BIT). Versión española. Madrid: TEA Ediciones.

Didden, R., R. H. J. Scholte, H. Korzilius, J. M. H. De Moor, A. Vermeulen, M. O’Reilly, and G. E. Lancioni. 2009. Cyberbullying Among Students with Intellectual and Developmental Disability in Special Education Settings. Developmental Neurorehabilitation 12 (3): 146-151. https://doi. org/10.1080/17518420902971356.

Fajardo, I., V. Ávila, G. Tavares, A. Ferrer, M. Gómez, and A. Hernández. 2014. EasyTo-Read Texts for Students with Intellectual Disability: Linguistic Factors Affecting Comprehension. Journal of Applied Research in Intellectual Disabilities 27: 212-25.

Fajardo, I., G. Tavares, V. Ávila, and A. Ferrer. 2013. Towards Text Simplification for Poor Readers with Intellectual Disability: When Do Connective Enhance Text Cohesion? Research in Developmental Disabilities 34: 1267-1279.

Gutiérrez, P., and A. Martorell. 2011. People with Intellectual Disability and ICTs. Comunicar 18 (36): 173-180. https://doi.org/10.3916/C36-2011-03-09.

Keil, F. C., and J. F. Kominsky. 2013. Missing Links in Middle School: Developing Use of Disciplinary Relatedness in Evaluating Internet Search Results. PloS ONE 8 (6): e67777. 
Livingstone, S., L. Haddon, A. Görzing, and K. Ólafsson. 2011. Risks and Safety on the Internet: The Perspective of European Children. Full findings. London (UK): EU Kids Online.

Lloyd, J., K. Moni, and A. Jobling. 2006. Breaking the Hype Cycle: Using the Computer Effectively with Learners with Intellectual Disabilities. Down Syndrome Research and Practice 9: 68-74.

Löfgren-Mårtenson, L. 2008. Love in Cyberspace: Swedish Young People with Intellectual Disabilities and the Internet. Scandinavian Journal of Disability Research 10 (2): 125-138.

Lough, E., and M. H. Fisher. 2016. Internet Use and Online Safety in Adults with Williams Syndrome. Journal of Intellectual Disability Research 60: 1020-1030.

Luckasson R., S. Borthwick-Duffy, W. H. E. Buntinx, D. L. Coulter, E. M. Craig, A. Reeve, R. L. Shalock, et al. 2002. Mental Retardation: Definition, Classification, and Systems of Supports. Washington (DC): American Association on Mental Retardation.

McConkey, R., and M. Smyth. 2003. Parental Perceptions of Risks with Older Teenagers who Have Severe Learning Difficulties Contrasted with the Young People's Views and Experiences. Children and Society 17 (1): 18-31. doi:10.1002/chi.725

Molin, M., E. Sorbring, and L. Löfgren-Martenson. 2015. Teachers' and Parents' Views on the Internet and Social Media Usage by Pupils with Intellectual Disabilities. Journal of Intellectual Disabilities $19 \quad$ (1): $22-33$. https://doi.org/10.1177/1744629514563558.

Morgan, M. F., M. Cuskelly, and K. B. Moni. 2011. Broadening the Conceptualization of Literacy in the Lives of Adults with Intellectual Disability. Research and Practice for Persons with Severe Disabilities 36 (3-4): 112-120.

Normand, C. L., and F. Sallafranque-St-Louis. 2016. Cybervictimization of Young People With an Intellectual or Developmental Disability: Risks Specific to Sexual Solicitation. Journal of Applied Research in Intellectual Disabilities 29 (2): 99-110. https://doi.org/10.1111/jar.12163.

Palmer, S. B., M. L. Wehmeyer, D. K. Davies, and S. E. Stock. 2012. Family Members' Reports of the Technology Use of Family Members with Intellectual and Developmental Disabilities. Journal of Intellectual Disability Research 56 (4): 402-414. 
Pérez, A., A. Potocki, M. Stadtler, M. Macedo-Rouet, J. Paul, L. Salmerón, and J. F. Rouet. 2018. Fostering Teenagers' Assessment of Information Reliability: Effects of a Classroom Intervention focused on Critical Source Dimensions. Learning and Instruction.

Perske, R. 1972. The Dignity of Risk. In The Principles of Normalization in Human Services, edited by W. Wolfensberger, B. Nirje, S. Olansky, R. Perske, and P. Roos, 194-200. Toronto, Canada: National Institute on Mental Retardation.

Rouet, J. F., C. Ros, A. Goumi, M. Macedo-Rouet, and J. Dinet. 2011. The Influence of Surface and Deep Cues on Primary and Secondary School Students' Assessment of Relevance in Web Menus. Learning and Instruction 21: 205-219.

Salmerón, L., R. Cerdán, and J. Naumann. 2015. How Adolescents Navigate Wikipedia to Answer Questions. Infancia y Aprendizaje: Journal for the Study of Education and Development 38: 435-471.

Salmerón, L., M. Gómez, and I. Fajardo. 2016a. How Students with Intellectual Disabilities Evaluate Recommendations from Internet Forums. Reading and Writing 29: 1653-1675.

Salmerón, L., Y. Kammerer, and P. García-Carrión. 2013. Searching the Web for Conflicting topics: Page and User Factors. Computers in Human Behavior 29: 2161-2171.

Salmerón, L., M. Macedo-Rouet, and J-F. Rouet. 2016b. Multiple Viewpoints Increase Students' Attention to Source Features in Social Question and Answer Forum Messages. Journal of the Association for Information Science and Technology 67: 2404-2419.

Seale, J. 2014. The Role of Supporters in Facilitating the Use of Technologies by Adolescents and Adults with Learning Disabilities: A Place for Positive RiskTaking? European Journal of Special Needs Education 29 (2): 220-236.

Seale, J. and D. Chadwick. 2017. How Does Risk Mediate the Ability of Adolescents and Adults with Intellectual and Developmental Disabilities to Live a Normal Life by Using the Internet? Cyberpsychology: Journal of Psychosocial Research on Cyberspace 11 (1): article 2. http://dx.doi.org/10.5817/CP2017-1-2

Sturm, J. M. 2012. An Enriched Writers' Workshop for Beginning Writers with Developmental Disabilities. Topics in Language Disorders 32 (4): 335-360.

Tavares, G., I. Fajardo, V. Ávila, L. Salmerón, and A. Ferrer. 2015. Who do You Refer to? How Young Students with Mild Intellectual Disability Confront Anaphoric 
Ambiguities in Texts and Sentences. Research in Developmental Disabilities 38: 108-124.

Van Nieuwenhuijzen, M., A. Vriens, M. Scheepmaker, M. Smit, and E. Porton. 2011. The Development of a Diagnostic Instrument to Measure Social Information Processing in Children With Mild To Borderline Intellectual Disabilities. Research in Developmental Disabilities 32 (1): 358-370.

World Health Organization. 2011. World report on disability. Malta: Author. 
Table 1. Examples of the coding system used to analyze the justifications of the selection task. These are literal translations from Spanish in which we tried to keep the original grammatical errors.

\begin{tabular}{ll}
\hline Topic relevance $\begin{array}{l}\text { justifications } \\
\text { jrustworthiness }\end{array}$ & $\begin{array}{l}\text { Because for me [the link label] better defines the information I } \\
\text { am looking for. }\end{array}$ \\
\cline { 2 - 2 } $\begin{array}{l}\text { justifications } \\
\text { Because it prevents you from the good and bad things of the diet. }\end{array}$ & $\begin{array}{l}\text { I think this is safer because it is the university and the social } \\
\text { networks are sometimes harmful. }\end{array}$ \\
\cline { 2 - 2 } $\begin{array}{l}\text { Out-of-focus } \\
\text { justifications }\end{array}$ & \begin{tabular}{l} 
Because it is a safety tip from a hospital. \\
\cline { 2 - 2 }
\end{tabular}
\end{tabular}


Figure 1. Sample question of the Ski-III questionnaire.

Query 1

You want to create an account in a new social network called Lingo. You have heard that it has better features than Tuenti, but that it could have security problems.

You have consulted on the Internet and have obtained 4 results. What page would you consult to inform you about the topic?

\section{Results}

Benefits and risks of Lingo

www.university-of-valencia.es/social-networks/lingo

Lingo is a virtual social network aimed at young people. We are going to analyze its risks and benefits, so that later...

Have fun without stopping with Lingo

www.easy-download.com/lingo

How do you access Lingo games? To see the games available in Lingo, use the search engine. You can filter by category (action, puzzle, sports ...

Lingo Tutorial for clumsy people

www.the-cybernaut-defender.com/ lingo-social-network

Quick guide that will help you to know at a glance all that a beginner needs to know about Lingo...

Advantages and disadvantages of Lingo

www.friendlyscreens.net/social-networks /lingo.shtm

During the last weeks, the social network Lingo has been ... Social networks, while having many advantages also have drawbacks ...

B-1 
Table 2. Percentage of participants that selected a particular page (high or low relevant topic, and high or low trustworthy page), for each topic.

\begin{tabular}{lcccc}
\cline { 2 - 4 } & Lingo & Facebook & Diet & Sunbath \\
\hline High topic relevance/ High page trustworthiness & $46.5 \%$ & $48.8 \%$ & $23.25 \%$ & $34.9 \%$ \\
\hline High topic relevance/ Low page trustworthiness & $27.9 \%$ & $16.3 \%$ & $34.9 \%$ & $27.9 \%$ \\
\hline Low topic relevance/ High page trustworthiness & $13.9 \%$ & $7.0 \%$ & $16.3 \%$ & $14.0 \%$ \\
\hline Low topic relevance/ Low page trustworthiness & $11.6 \%$ & $27.9 \%$ & $25.5 \%$ & $23.2 \%$ \\
\hline
\end{tabular}


Table 3. Number of words for the justification regarding the selection of a particular page (first row), and number of students whom used each type of justification, for each of the four topics (second to fourth rows).

\begin{tabular}{lrrrr}
\cline { 2 - 4 } & Lingo & Facebook & Diet & Sunbath \\
\hline Justification length (words) & $7.7(4.2)$ & $9.3(5.5)$ & $8.3(5.0)$ & $7.6(4.2)$ \\
\hline Topic justification & $15(34.9 \%)$ & $11(25.6 \%)$ & $11(25.6 \%)$ & $11(25.6 \%)$ \\
\hline Trust justification & $1(2.3 \%)$ & $3(7.0 \%)$ & $5(11.6 \%)$ & $2(4.6 \%)$ \\
\hline Out-of-focus justification & $27(62.8 \%)$ & $29(67.44 \%)$ & $27(62.8 \%)$ & $30(69.8 \%)$ \\
\hline
\end{tabular}

